\title{
Análisis Elemental de las Propiedades Morfológicas, Físicas y Químicas de Dos Instrumentos Rotatorios Protaper Universal
}

\author{
Elemental Analysis of the Morphological, Physical and Chemical \\ Properties of Two Protaper Universal Rotary Instruments
}

\begin{abstract}
José Roberto Gutiérrez-Camacho'; Norma Verónica Zavala-Alonso²; Carlos Bermúdez-Jiménez³; Luis Alejandro Aguilera-Galaviz ${ }^{3}$; Margarita de la Luz Martínez-Fierro4; Víctor Baltazar-Hernández ${ }^{5}$ \& Cesar Gaitán-Fonseca ${ }^{3}$
\end{abstract}

GUTIÉRREZ-CAMACHO, J. R.; ZAVALA-ALONSO, N. V.; BERMÚDEZ-JIMÉNEZ, C.; AGUILERA-GALAVIZ, L. A.; MARTÍNEZ-FIERRO, M. L.; BALTAZAR-HERNÁNDEZ, V. \& GAITÁN-FONSECA, C. Análisis elemental de las propiedades morfológicas, físicas y químicas de dos instrumentos rotatorios protaper universal. Int. J. Odontostomat., 15(2):381-386, 2021.

RESUMEN: Los instrumentos rotatorios utilizados en el tratamiento de conductos dentales necesitan cumplir con ciertas características físicas que le confieran un uso correcto, adecuado y seguro para su uso clínico. En el mercado comercial existen una serie de instrumentos importados que dejan en duda la autenticidad de estos debido a sus bajos costos. En este estudio evaluamos física, química y morfológicamente dos kits de instrumentos rotatorios Protaper universal (Dentsply) mediante análisis de MEB, EDX, microdureza y EDS. Uno de los kits (grupo 1) se adquirió directamente de la casa comercial y otro kit importado que a simple vista no parecía cumplir con los estándares de calidad de un correcto empaquetado (grupo 2). En el análisis de MEB se observaron características morfológicas muy diferentes entre ambos kits, el grupo 2 presentó diversas irregularidades en la superficie de las limas, sin embargo, en el EDS no se encontró diferencia alguna. En cuanto al análisis de microdureza se observó una estadística estadísticamente significativa y en el EDS se observaron mayores picos de intensidad en cuanto a la aleación de $\mathrm{Ni}$-Ti en el grupo 2. Estos resultados sugieren que existen instrumentos rotatorios importados que a pesar de su bajo costo pueden presentar ciertas características muy similares a los kits auténticos, sin embargo, física y químicamente pueden resultar en un riesgo para su uso clínico debido a la diferencia entre estos.

PALABRAS CLAVE: limas rotatorias, endodoncia, microdureza, protaper, composición elemental, espectroscopia de energía dispersiva.

\section{INTRODUCCIÓN}

En la actualidad el tratamiento de conductos radiculares ha generado un gran avance científico y tecnológico derivado de la variabilidad en materiales principalmente $\mathrm{Ni}$-Ti para el desarrollo de esta técnica clínica (Zupanc et al., 2018; Bhatt \& Rajkumar, 2019; Rubio et al., 2019). Diversas casas comerciales se han preocupado por generar instrumentos endodónticos manuales, rotatorios, reciprocantes y adaptativos que de acuerdo a cada casa comercial utilizan diferentes especificaciones técnicas y equipos para su uso. Además de evaluar protocolos para aquellos casos donde se pueda presentar fractura por fatiga cíclica o eva-

\footnotetext{
${ }^{1}$ Estudiante del Doctorado con orientación en Medicina Molecular, Laboratorio de Medicina Molecular, Unidad Académica de Medicina Humana, Área de Ciencias de la Salud, Universidad Autónoma de Zacatecas "Francisco García Salinas", Zacatecas, Zac. México.

2 Docente-Investigador, Laboratorio del Doctorado de Ciencias Odontológicas, Universidad Autónoma de San Luis Potosí, San Luis Potosí, S.L.P. México.

${ }^{3}$ Docente-Investigador, Unidad Académica de Odontología, Universidad Autónoma de Zacatecas "Francisco García Salinas", Zacatecas, Zac. México.

${ }^{4}$ Laboratorio de Medicina Molecular, Unidad Académica de Medicina Humana, Área de Ciencias de la Salud, Universidad Autónoma de Zacatecas "Francisco García Salinas", Zacatecas, Zac. México.

${ }^{5}$ Docente-Investigador, Doctorado en Ingeniería y Ciencia de Materiales, Universidad Autónoma de Zacatecas "Francisco García Salinas",
} Zacatecas, Zac.México. 
GUTIÉRREZ-CAMACHO, J. R.; ZAVALA-ALONSO, N. V.; BERMÚDEZ-JIMÉNEZ, C.; AGUILERA-GALAVIZ, L. A.; MARTíNEZ-FIERRO, M. L.; BALTAZAR-HERNÁNDEZ, V. \& GAITÁN-FONSECA, C. Análisis elemental de las propiedades morfológicas, físicas y químicas de dos instrumentos rotatorios protaper universal. Int. J. Odontostomat., 15(2):381-386, 2021

luar sistemas endodónticos después de haber sido sometidos a un retratamiento endodóntico (Cassol et al., 2020; Özyürek \& Demiryürek, 2020). En particular, los sistemas endodónticos rotatorios se han mantenido en el mercado comercial por su variabilidad, técnicas de uso, amplia disposición, y en algunos casos costos accesibles. Pero además requiere de una adecuada fase de entrenamiento entre los estudiantes y especialistas que prefieren utilizar dientes artificiales en un $60 \%$ y dientes confeccionados por microtomografía computarizada (Micro-CT) del complejo anatómico de dientes naturales en un $70 \%$, para su uso pertinente tratando de minimizar los riesgos durante su empleo (Gancedo-Caravia et al., 2020).

Sin embargo, en esta versatilidad y auge comercial existe una interrogante sobre la reutilización de los sistemas de instrumentación endodóntica, cuando de manera general las casas comerciales dan las recomendaciones e indicaciones de un solo uso clínico. Buchanan \& Warren (2019), en su estudio menciona las ventajas de un solo uso de limas endodónticas como: dificultad para una adecuada limpieza de los instrumentos, el riesgo de transmitir enfermedades infecciosas, la trasmisión potencial de prion, fatiga y corrosión de los instrumentos, pero además describe los argumentos para la reutilización de instrumentos: eliminación $100 \%$ del debris de la superficie de los instrumentos por limpieza en laboratorio, la ausencia de proteínas prion en pulpas dentales de individuos con diagnóstico de enfermedad de Creutzfeldt-Jakob, el costo incrementado en protocolos de un solo uso, adecuados métodos de esterilización y desinfección de instrumentos (Maniglia-Ferreira et al., 2017; Buchanan \& Warren). En ese sentido un sistema que tiene amplia aceptación global es Protaper Universal (PTU, Dentsply, USA), que tienen un adecuado desempeño en su fabricación y la indicación de un uso clínico en las recomendaciones de su propia casa comercial (Montenegro-Santillán et al., 2013).

Logsdon et al. (2020) realizaron una encuesta a miembros activos de la American Association of Endodontists (AAE), en la cual $98,3 \%$ usa los instrumentos de Nickel-Titanio (Ni-Ti), 56,9\% tiende a usar productos de la casa comercial Denstply y el $74 \%$ tiende a reutilizar los instrumentos. La reutilización de sistemas $\mathrm{Ni}-\mathrm{Ti}$, ha permitido seguir innovando en la creación de nuevos sistemas comerciales, a la evaluación de las superficies de los sistemas actuales (Bennett et al., 2017), y desafortunadamente también a la manufactura de instrumentos a costos bajos por encima del promedio y que se pueden adquirir a bajos costos como productos de importación. Aunque comercialmente pre- sentan las mismas características de empaque, existen diferencias que pueden ser identificables por especialistas en el ramo. Particularmente en este artículo se describen algunas de las diferencias que se evidencian al momento de comparar dos estuches comerciales de PTU: diferencia en la calidad de impresión del etiquetado comercial, no presencia de holograma que certifica la autenticidad del producto, una rotación no uniforme y fuera de su eje central en su uso clínico y finalmente ausencia del pulido final de la superficie del instrumento. Por lo que el objetivo del presente estudio fue evaluar características físicas, químicas y morfológicas de dos sistemas de PTU, Dentsply.

\section{MATERIAL Y MÉTODO}

Se realizó un estudio experimental, descriptivo y analítico que incluyó la incorporación de dos kits de limas Protaper universal (Dentsply). Grupo 1 (PTU, N de lote: 1182039, fecha de fabricación 2018), Grupo 2 (PTU, $N^{\circ}$ de lote: 5341450 , fecha de fabricación 2014). Cada grupo estuvo constituido por 10 limas. A estos dos sistemas se realizaron las siguientes pruebas de evaluación:

a) Microscopía electrónica de barrido y EDX. Los grupos experimentales fueron recubiertos con oro para ser analizados por medio de microscopía electrónica de barrido (JSM-6510; Jeol Ltd., Tokio, Japón) para la obtención de imágenes. Además, se realizó un análisis EDX por medio de espectroscopia de dispersión de rayos X (X-MaxN Oxford Instruments, Abingdon, Reino Unido) a través del software Aztec Energy EDS Analysis System. Se obtuvieron 12 imágenes de las superficies de los instrumentos endodónticos en condiciones de cámara de alto vacío y utilizando un voltaje de aceleración de $15 \mathrm{kV}$. Las composiciones elementales de $\mathrm{Ni}-\mathrm{Ti}$ de las superficies de las muestras con contraste del número atómico medio se analizaron mediante el análisis de puntos EDX sobre la parte activa de los instrumentos (Al Jabbari et al., 2016).

b) Microdureza. Para evaluar la resistencia de las superficies de cada uno de los grupos se realizó análisis de microdureza (Vickers y Rockwell) con un peso inferior a 1.961 Milinewtons $(\mathrm{mN})$. Los sistemas endodónticos de manera inicial se encapsularon en lutita y posteriormente se desbastó la capsula, finalmente se sometió a proceso de pulimiento en una pulidora metalográfica CIENTEC con alúmina de $300 \mu \mathrm{m}$. Estas pruebas se realizaron a temperatura ambiente. La 
GUTIÉRREZ-CAMACHO, J. R.; ZAVALA-ALONSO, N. V.; BERMÚDEZ-JIMÉNEZ, C.; AGUILERA-GALAVIZ, L. A.; MARTíNEZ-FIERRO, M. L.; BALTAZAR-HERNÁNDEZ, V. \& GAITÁN-FONSECA, C. Análisis elemental de las propiedades morfológicas, físicas y químicas de dos instrumentos rotatorios protaper universal. Int. J. Odontostomat., 15(2):381-386, 2021

microdureza permitió caracterizar la resistencia a la penetración sobre la superficie de los grupos 1 y 2 (Kuhn et al., 2001).

c) Espectroscopía Dispersiva de Energía (EDS). En el orden de analizar la fase de transformación, la técnica EDS fue conducida por medio de radiación Cu-Ka en un difractómetro D8 Advanced-ECO Bruker ${ }^{\mathrm{TM}}$ a temperatura ambiente. Se configuro en el instrumento un modo de escaneo continuo en un modo acoplado de dos thetatheta, en un rango de 2 theta de $35-120$ grados, un tamaño de paso de $0.01^{\circ}$.

d) Análisis estadístico. Los datos se analizaron en primer lugar utilizando la prueba de Shapiro-Wilk para verificar la suposición de normalidad. La significancia estadística se determinó utilizando la prueba t de Student para muestras no relacionadas mediante el software GraphPad Prism versión 5.03 (San Diego, CA). Se consideró una $p \leq 0.05$ como estadísticamente significativa.

\section{RESULTADOS}

a) Análisis del MEB y EDX. Las microfotografías microscópicas de barrido electrónico revelaron en el gru- po 1 (Figs.1A, 1B y 1C) superficies uniformes, pulidas y sin irregularidades que puedan comprometer la resistencia del instrumento. En el grupo 2 (Figs. 1D, 1E y 1F) se observa ausencia de pulido y la presencia de múltiples irregularidades en toda la superficie de los instrumentos.

En la Tabla I se describen los porcentajes de $\mathrm{Ni}$ Ti de los grupos experimentales que se obtuvieron por medio del análisis EDX. Se observa que los instrumentos del grupo 1 tienen un porcentaje mayor respecto a los instrumentos del grupo 2, sin embargo, no existe una diferencia estadísticamente significativa $(p>0.05)$.

b) Análisis de Microdureza. En la Tabla II se observan los valores de la microdureza de Vickers (HV) y Rockwell $(\mathrm{Hrc})$ de las superficies de los instrumentos. El grupo 2 presenta valores mayores en comparación con el grupo 1 observándose una diferencia estadísticamente significativa $(p<0.05)$.

c) Análisis de Espectroscopía Dispersiva de Energía (EDS). En el análisis de Espectroscopia Dispersiva de Energía (EDS) se observa en la Figura 2 que el grupo 2 presenta un mayor pico (>6000 a.u.) de intensidad con relación a la aleación $\mathrm{Ni}$-Ti y a los elementos Titanio y Níquel comparado con el grupo 1 (2300 a.u.).
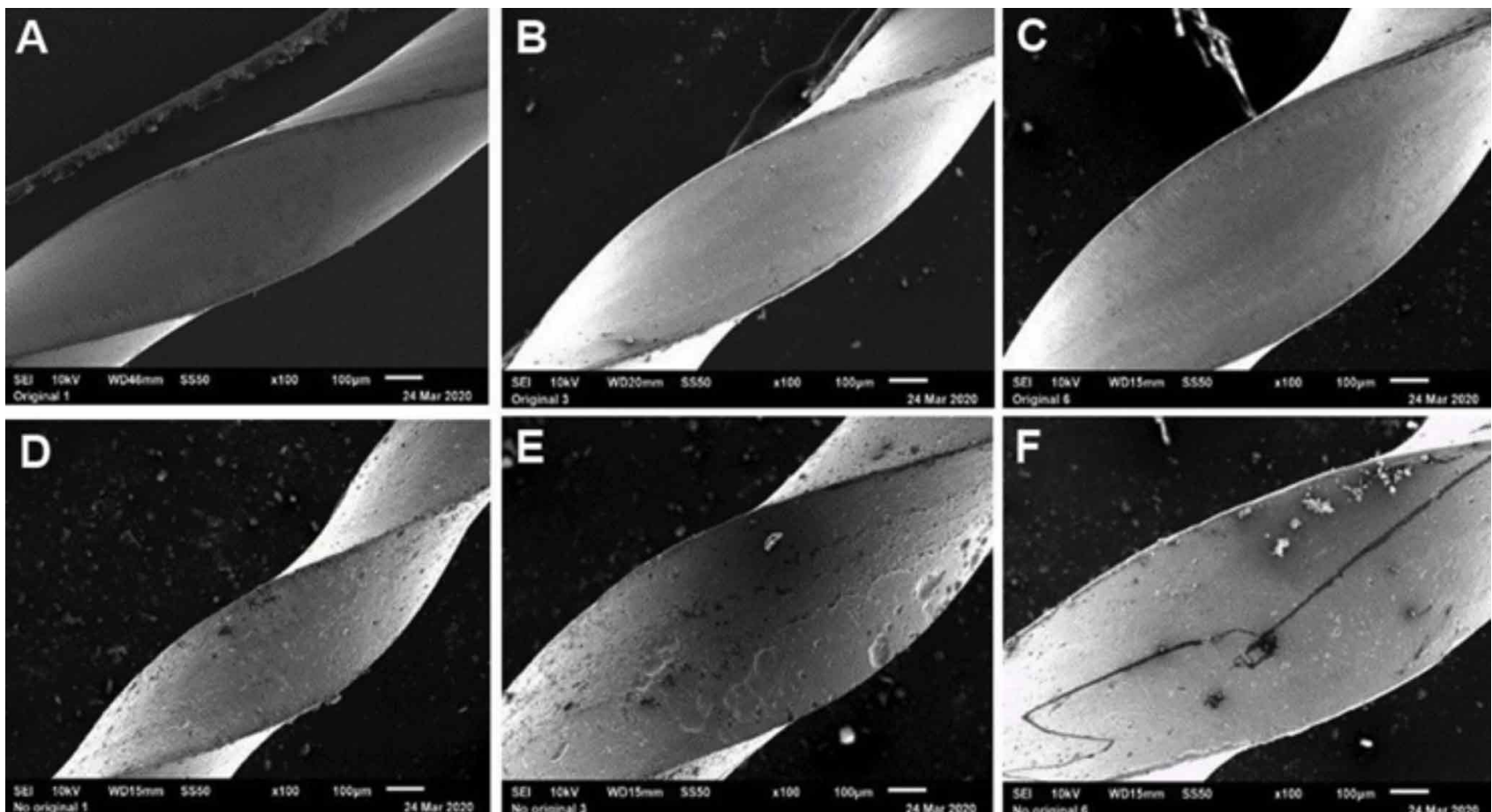

Fig. 1. Imágenes MEB. En las muestras A, B, C, observadas a 100x imágenes del Grupo 1 en donde se observa una notable diferencia sobre el tratamiento de la superficie de los instrumentos. Las muestras D, E y F corresponden al Grupo 2 se puede apreciar la ausencia del pulido de la superficie, así como irregularidades sobre los instrumentos. 
GUTIÉRREZ-CAMACHO, J. R.; ZAVALA-ALONSO, N. V.; BERMÚDEZ-JIMÉNEZ, C.; AGUILERA-GALAVIZ, L. A.; MARTÍNEZ-FIERRO, M. L.; BALTAZAR-HERNÁNDEZ, V. \& GAITÁN-FONSECA, C. Análisis elemental de las propiedades morfológicas, físicas y químicas de dos instrumentos rotatorios protaper universal. Int. J. Odontostomat., 15(2):381-386, 2021.

Tabla I. Promedio (X) y desviación estándar (D.E.) de los dos grupos de instrumentos rotatorios en cuanto al porcentaje de nickel $(\mathrm{Ni})$ y titanio $(\mathrm{Ti})$ obtenidos mediante el análisis EDX.

\begin{tabular}{lcc}
\hline & $\mathrm{Ni} \%(\mathrm{X} \pm \mathrm{D} . \mathrm{E})$. & $\mathrm{Ti} \%(\mathrm{X} \pm \mathrm{D} . \mathrm{E})$. \\
\hline Grupo 1 & $52,98 \pm 6,39$ & $36,28 \pm 1,48$ \\
Grupo 2 & $50,35 \pm 7,84$ & $35,39 \pm 1,58$ \\
Valor $p^{*}$ & $>0,05$ & $>0,05$
\end{tabular}

*Estadísticamente significativa $p<0.05$.

Tabla II. Promedio (X) y desviación estándar (D.E.) del análisis de Vickers (HV) y Rockwell (Hrc) en los dos grupos de estudio.

\begin{tabular}{lcc}
\hline & $\mathrm{HV}(\mathrm{X} \pm \mathrm{D} . \mathrm{E})$. & $\mathrm{Hrc}(\mathrm{X} \pm \mathrm{D} . \mathrm{E})$. \\
\hline Grupo 1 & $290 ., 02 \pm 0,49$ & $270,50 \pm 1,83$ \\
Grupo 2 & $310,48 \pm 0,67$ & $310,60 \pm 0,78$ \\
Valor $p$ & $<0,05$ & $<0.05$ \\
\hline
\end{tabular}

*Estadísticamente significativa $p<0.05$.

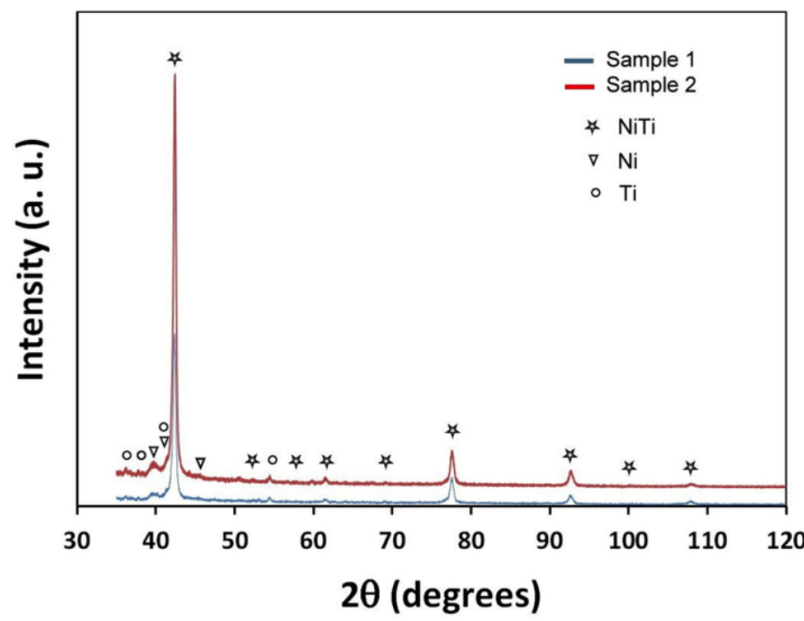

Fig. 2. Análisis de Espectroscopia Dispersiva de Energía (EDS). Se observa que el grupo 2 expresa un pico mayor de intensidad en la aleación $\mathrm{Ni}$-Ti, respecto del grupo 1.

\section{DISCUSIÓN}

En este estudio se evaluaron por medio de MEB, análisis EDX, dureza Vickers y Rockwell y EDS, la composición metalúrgica y propiedades físicas de dos kits de Protaper universal (grupo 1 y 2) con diferente lotificación. Los instrumentos del grupo 1 se consideraron para este estudio como originales y el grupo 2 como no originales. Esto de acuerdo con Castro-Núñez et al. (2016) y derivado de la revisión de estos dos lotes en sus empaques comerciales y a la vez en su funcionamiento rotativo durante su montaje en el motor rotatorio de acuerdo con sus especificaciones de uso (Castro-Núñez et al.).
Durante la instrumentación en el conducto radicular los sistemas rotatorios son sometidos a cargas torsionales y de fatiga que pueden conllevar al desprendimiento del instrumento. Derivado de esta situación, es de relevancia poder evaluar las características metalúrgicas y físicas de los instrumentos para evaluar su calidad y eficiencia clínica (FernándezPazos et al., 2018). En este sentido, Weyh \& Ray (2020) evaluaron diferentes sistemas comerciales $\mathrm{Ni}$ Ti realizando análisis EDX y test de fatiga cíclica, demostró que el porcentaje de Ni-Ti fue muy similar entre todos los grupos evaluados.

La disparidad en el patrón de fatiga cíclica y el diseño de la punta en las imágenes al MEB determina que el clínico no debe esperar un comportamiento similar en todos los sistemas (Weyh \& Ray). Los sistemas rotatorios $\mathrm{Ni}$-Ti pueden ser fabricados por dos procesos: electropulido y mecanizado por descarga eléctrica (EDM). Estos procesos les confieren propiedades para poder ser utilizados en la gran variabilidad anatómica del conducto radicular. En nuestros resultados observados al MEB se puede observar que el grupo 1 PTU presenta este proceso de electropulido, mismo que se observa deficiente en los instrumentos del grupo 2. En la Figura 1C se observan algunas líneas sobre la superficie del instrumento (Purayil et al., 2018; Gavini et al., 2018). Sin embargo, al comparar mediante el análisis de EDX el porcentaje de NiTi de ambos grupos, no se observó una diferencia estadísticamente significativa ( $p>0.05$ ) lo cual nos hace suponer que a pesar de que los instrumentos no pasaron por el mismo control de calidad en cuanto al pulido, la composición es muy similar entre ambos grupos (Hamdy et al., 2019).

En relación con el análisis de microdureza y a lo reportado por Elnaghy \& Elsaka (2014), en su estudio evaluaron la microdureza de limas Protaper Next (PTN) y presento un valor de $351.95 \mathrm{HV}$, y que comparado con el grupo 2 de PTU en nuestro estudio fue de $310.48 \mathrm{HV}$. Este incremento en la microdureza está asociado a la tecnología M-Wire y diseño rectangular que presenta PTN (Elnaghy \& Elsaka). Clínicamente estos valores nos ayudaran a elegir el sistema rotatorio para la instrumentación de conductos estrechos, con curvaturas o presencia de calcificaciones.

En el Análisis de Espectroscopia Dispersiva de Energía (EDS), se evaluó el grado de transformación de los instrumentos. Para este estudio los sistemas no fueron sometidos a trabajo clínico. En este sentido el grupo 2 presentó mejores valores de intensidad en 
relación a la aleación NiTi y los elementos $\mathrm{Ni} \mathrm{y} \mathrm{Ti} \mathrm{por}$ separado respecto al grupo 1 (Üreyen Kaya et al., 2019). De acuerdo con lo reportado por Prasad et al. (2014), mencionan que el porcentaje de Nitinol en los sistemas rotatorios contiene aproximadamente $56 \%$ (Ni) y $44 \%$ (Ti). Los instrumentos rotatorios al entrar en contacto con soluciones irrigantes como $\mathrm{NaOCl}$ y EDTA pueden disminuir su porcentaje de Nitinol e incrementar el porcentaje de elementos $\mathrm{Cl}$ y $\mathrm{Na}$ en la superficie. Esto puede contribuir a la presencia de irregularidades, durabilidad y seguridad en los conductos radiculares (Prasad et al.).

\section{CONCLUSIÓN}

La evaluación in vitro de los instrumentos endodónticos se ha incrementado debido a la gran demanda y oferta comercial que existe, este tipo de análisis nos permite conocer de manera más adecuada el comportamiento probable de estos instrumentos para una condición clínica real. Por lo que es necesario continuar con este tipo de estudios para evaluar materiales de importación que a simple vista parecen iguales pero que clínicamente pueden tener efectos no deseados.

GUTIÉRREZ-CAMACHO, J. R.; ZAVALA-ALONSO, N. V.; BERMÚDEZ-JIMÉNEZ, C.; AGUILERA-GALAVIZ, L. A.; MARTÍNEZ-FIERRO, M. L.; BALTAZARHERNÁNDEZ, V. \& GAITÁN-FONSECA, C. Elemental analysis of the morphological, physical and chemical properties of two protaper universal rotary instruments. Int. J. Odontostomat., 15(2):381-386, 2021.

ABSTRACT: Rotatory files are instruments used in the treatment of dental canals roots. These instruments need to comply with certain physical characteristics for a correct, adequate and safe use for clinical use. In the commercial market there are a series of imported instruments that cast doubt on their authenticity due to their low costs. In this study we physically, chemically and morphologically evaluated two Protaper universal rotary instrument kits (Dentsply) using SEM, EDX, microhardness and EDS analysis. One of the kits (group 1) was purchased directly from the commercial house and another imported kit that a simple view did not seem to meet the quality standards of a correct packaging (group 2). In the SEM analysis, very different morphological characteristics were observed between both kits, group 2 presented various irregularities on the surface of the files, however, no difference was found in the EDS. Regarding the microhardness analysis, a statistically significant statistic was observed and in the EDS, higher intensity peaks were observed in terms of the $\mathrm{Ni}$-Ti alloy in group 2 . These results suggest that there are imported rotary instruments that despite their low cost can present characteristics very similar to authentic kits, however, physically and chemically they can result in a risk for their clinical use due to the difference between them.

KEY WORDS: rotary instruments, endodontics, microhardness, protaper, elemental composition, energy dispersive spectroscopy.

\section{REFERENCIAS BIBLIOGRÁFICAS}

Al Jabbari, Y. S.; Koutsoukis, T.; Al Hadlaq, S.; Berzins, D. W. \& Zinelis, S. Surface and cross-sectional characterization of titanium-nitride coated nickel-titanium endodontic files. J. Dent. Sci., 11(1):48-53, 2016.

Bennett, J.; Chung, K. H.; Fong, H.; Johnson, J. \& Paranjpe, A. Analysis of Surface Characteristics of ProTaper Universal and ProTaper Next instruments by scanning electron microscopy. J. Clin. Exp. Dent., 9(7):e879-85, 2017.

Bhatt, A. \& Rajkumar, B. A comparative evaluation of cyclic fatigue resistance for different endodontic NiTi rotary files: An invitro study. J. Oral Biol. Craniofac. Res., 9(2):119-21, 2019.

Buchanan, G. D. \& Warren, N. Single-use of endodontic hand files: perceptions and practise. J. Infect. Prev., 20(1):32-6, 2019.

Cassol, L. G.; Kowalczuck, A.; Carneiro, E.; Westphalen, V. P. D.; Laurindo, C. A. H. \& da Silva Neto, U. X. Evaluation of fluoride and sodium hypochlorite solutions during the electrochemical dissolution of conventional $\mathrm{NiTi}$ instruments and Gold thermomechanically treated $\mathrm{NiTi}$ instruments. Int. Endod. J., 53(4):513-8, 2020.

Castro-Núñez, G.; Escalante-Otárola, W.; Scapin Viola, K.; Coaguila-Llerena, H.; Faria, G. \& Kuga, M. C. Comparación del sistema ProTaper Universal con homólogo adulterado: estudio descriptivo. Rev. Estomatol. Herediana, 26(3):184-90, 2016.

Elnaghy, A. M. \& Elsaka, S. E. Assessment of the mechanical properties of ProTaper Next Nickel-titanium rotary files. J. Endod., 40(11):1830-4, 2014.

Fernández-Pazos, G.; Martín-Biedma, B.; Varela-Patiño, P.; RuízPiñón, M. \& Castelo-Baz, P. Fracture and deformation of ProTaper Next instruments after clinical use. J. Clin. Exp. Dent., 10(11):e1091-5, 2018.

Gancedo-Caravia, L.; Bascones, J.; García-Barbero, E. \& Arias, A. Suitability of different tooth replicas for endodontic training: perceptions and detection of common errors in the performance of postgraduate students. Int. Endod. J., 53(4):562-72, 2020.

Gavini, G.; Dos Santos, M.; Caldeira, C. L.; de Lima Machado, M. E.; Freire, L. G.; Iglecias, E. F.; Peters, O. A. \& Candeiro, G. T. M. Nickel-titanium instruments in endodontics: a concise review of the state of the art. Braz. Oral Res., 32(Suppl. 1):e67, 2018.

Hamdy, T. M.; Galal, M.; Ismail, A. G. \& Abdelraouf, R. M. Evaluation of flexibility, microstructure and elemental analysis of some contemporary nickel-titanium rotary instruments. Open Access Maced. J. Med. Sci., 7(21):3647-54, 2019.

Kuhn, G.; Tavernier, B. \& Jordan, L. Influence of structure on nickeltitanium endodontic instruments failure. J. Endod., 27(8):51620, 2001. 
GUTIÉRREZ-CAMACHO, J. R.; ZAVALA-ALONSO, N. V.; BERMÚDEZ-JIMÉNEZ, C.; AGUILERA-GALAVIZ, L. A.; MARTíNEZ-FIERRO, M. L.; BALTAZAR-HERNÁNDEZ, V. \& GAITÁN-FONSECA, C. Análisis elemental de las propiedades morfológicas, físicas y químicas de dos instrumentos rotatorios protaper universal. Int. J. Odontostomat., 15(2):381-386, 2021.

Logsdon, J.; Dunlap, C.; Arias, A.; Scott, R. \& Peters, O. A. Current trends in use and reuse of nickel-titanium engine-driven instruments: a survey of endodontists in the United States. J. Endod., 46(3):391-6, 2020.

Maniglia-Ferreira, C.; de Almeida Gomes, F.; Ximenes, T.; Neto, M. A. T.; Arruda, T. E.; Ribamar, G. G. \& Herculano, L. F. G. Influence of reuse and cervical preflaring on the fracture strength of reciprocating instruments. Eur. J. Dent., 11(1):41-7, 2017.

Montenegro-Santillán, R.; Alegre-Domingo, T.; Faus-Matoses, V. \& Faus-Llácer, V. An in vitro comparison of cyclic fatigue resistance of ProTaper universal and GT series X files. Med. Oral Patol. Oral Cir. Bucal, 18(3):e533-6, 2013.

Özyürek, T. \& Demiryürek, E. Ö. Surface deformation of several nickel-titanium rotary instruments after removal of the root canal filling materials: a scanning electron microscope evaluation. Odontology, 108(2):213-21, 2020.

Prasad, P. S.; Sam, J. E.; Kumar, A. \& Kannan. The effect of $5 \%$ sodium hypochlorite, $17 \%$ EDTA and triphala on two different rotary $\mathrm{Ni}-\mathrm{Ti}$ instruments: An AFM and EDS analysis. J. Conserv. Dent., 17(5):462-6, 2014.

Purayil, T. P.; Ballal, N. V. \& Ginjupalli, K. Comparative evaluation of surface defects in single file rotary systems before and after instrumentation in curved root canals. Eur. J. Prosthodont. Restor. Dent., 26(3):130-5, 2018.

Rubio, J.; Zarzosa, J. I. \& Pallarés, A. A comparative study of cyclic fatigue of 10 different types of endodontic instruments: an in vitro study. Acta Stomatol. Croat., 53(1):28-36, 2019.

Üreyen Kaya, B.; Erik, C. E. \& Kiraz, G. Atomic force microscopy and energy dispersive X-ray spectrophotometry analysis of reciprocating and continuous rotary nickel-titanium instruments following root canal retreatment. Microsc. Res. Tech., 82(7):115764, 2019.

Weyh, D. J. \& Ray, J. J. Cyclic fatigue resistance and metallurgic comparison of rotary endodontic file systems. Gen. Dent., 68(1):36-9, 2020.

Zupanc, J.; Vahdat-Pajouh, N. \& Schäfer, E. New thermomechanically treated NiTi alloys - a review. Int. Endod. J., 51(10):1088-103, 2018.
Dirección para correspondencia:

César Gaitán-Fonseca

Unidad Académica de Odontología

Universidad Autónoma de Zacatecas

"Francisco García Salinas"

CP 98600, Zacatecas, Zac.

MEXICO

E-mail: cgaitan@uaz.edu.mx 